Insight Pharmaceutical Sciences 2 (1): 1-7, 2012

ISSN 2040-705X / DOI: 10.5567/IPHARMA-IK.2012.1.7

(C) 2012 Insight Knowledge, UK

\title{
Potentiometric and HPLC Methods for the Determination of Meloxicam in Bulk and Drug Formulations
}

\author{
${ }^{1}$ Mohammed A. El-Ries, ${ }^{2}$ Farida El-Dars, ${ }^{1}$ Fekria M. Abou Attia, ${ }^{2}$ A.B. Farag and ${ }^{1}$ Ayat M. Abd El-Hamed \\ ${ }^{1}$ National Organization for Drug Control and Research (NODCAR), 35521, Giza, Egypt \\ ${ }^{2}$ Department of Chemistry, Faculty of Science, Helwan University, Helwan, 11795, Egypt
}

\begin{abstract}
Purpose: The present study describes highly sensitive, precise, economical and quick potentiometric and HPLC methods for the determination of meloxicam in pharmaceutical formulations. Methods: The first method is a potentiometric one (I) where the titration of the drug with N-Bromosuccinimide (NBS) and N-Bromophethalimide (NBP)is involved in acid medium and the end point is determined using a platinum indicator electrode. The second one is a sensitive and selective HPLC method (II). The method is applied using a mobile phase consisted of acetonitrile: $\mathrm{H}_{2} \mathrm{O}$ :phosphoric acid (70: $\left.30: 0.1, \mathrm{v} / \mathrm{v} / \mathrm{v}\right)$ on a $150 \times 4.6 \mathrm{~mm} \mathrm{C}_{18}$ column and detection wavelength at $360 \mathrm{~nm}$. The intra-day, inter-day precision and accuracy, recovery, stability, reproducibility and sensitivity of the proposed methods are presented. Results: Method (I) is valid over the concentration range $0.176-1.406 \mathrm{mg}$ Mel with mean percentage accuracy 100.26 and $100.08 \%$ for NBS and NBP, respectively. The content of the mobile phase in method (II) is simple in preparation, does not depend on adjusting $\mathrm{pH}$ and is found to be fast( single sharp and symmetric peak is obtained after retention time $\sim 2.1 \mathrm{~min}$ ) at room temperature. In addition, this system provides a linear response over the concentration range 5-200 $\mu \mathrm{g} \mathrm{mL}^{-1} \mathrm{Mel}$, with mean percentage accuracy of $99.41 \%$. The intra-and inter-day relative standard deviation over the entire concentration range is less than $1.5 \%$. Conclusion: The proposed methods are successfully applied to pharmaceutical dosage forms and the results are in agreement with those given by the official method.
\end{abstract}

Key words: Meloxicam, Potentiometry, N-Bromosuccinimide, N- Bromophethalimide, HPLC, Commercial formulations

\section{INTRODUCTION}

Meloxicam(Mel)(4-hydroxy-2-methyl-N-\{5-methyl-2thiazolyl\}-2-H-1, 2-benzothiazine 3-carboxamide 1,1dioxide) (Fig. 1) is a Non-steroidal Anti-inflammatory Drug (NSAD) of the oxicam class. The drug is effective in the treatment of rheumatoid, arthritis, osteoarthritis and related conditions (Martindale, 2002).

A review of analytical techniques for determination of oxicams, nimesulide and nabumetone covers the time period from 1990 to 2008 , in which analytical methods

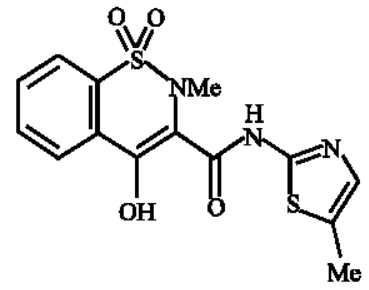

Fig. 1: Structure of meloxicam including all types of chromatographic, spectrophotometric and voltammetric techniques are reported (Jan and Starek, 2009). The literature survey reveals that HPLC methods (Yuan, Chen and Zhong, 2007; Desai and Amin, 2008; Sinha et al., 2009; Bandarkar and Vavia, 2009; Lee et al., 2009; Eroglu et al., 2010; Mahmood et al., 2010; Radia and Moulay-Hassan, 2010), are the most commonly used methods for the estimation of $\mathrm{Mel}$ in human plasma and pharmaceutical forms. Other analytical techniques have been reported for the analysis of Meloxicam in formulations and biological samples, such as spectrophotometry (Liu et al., 2005; Al-Nimer and Ali, 2009; Huazhen et al., 2009; Al-Kindy et al., 2010); Thanh et al., 2010); Dhandapani et al., 2010), voltammetry (Cheng et al., 2006; Farhadi and Karimpour, 2007) and polarography (Acuna et al., 2004).

While HPLC methods continue to play a major role in the development of analytical procedures applying in laboratory for the determination of pharmaceutical

Corresponding Author: Mohammed A. El-Ries, National Organization for Drug Control and Research (NODCAR), 35521, Giza, Egypt 
compounds, NBS has been widely used as an oxidizing agent in acidic media (Kumar and Inrasenan, 1988), Titrimetric methods for the determination of some sulpha drugs using N-bromophthalimide and N-bromosaccharin and more extensively, it has been used in the determination of a vast number of compounds, especially those of pharmaceutical interest.

Up till now, there has been no publication on determination of Meloxicam using potentiometric titration with N-halo compounds. In this study, in addition to HPLC method, we have developed a selective potentiometric titration method based on using NBS and NBP as oxidizing agents. This reaction is studied in order to intercept the mechanism and to understand the redox chemistry of NBS and NBP with Mel in such media. The proposed methods are simple, accurate and reproducible indicating their suitability for routine determination of $\mathrm{Mel}$ in bulk and dosage forms.

\section{MATERIALS AND METHODS}

Meloxicam is obtained from (Delta Pharma Company) 99.95\% purity (USP, 2009) and is used as received. Preparations containing Meloxicam (Melocam tablets labeled to contain 15 and $7.5 \mathrm{mg} /$ tablet, Amoun Pharma, Cairo, Egypt) and (Mexicam tablets labeled to contain 15 and $7.5 \mathrm{mg}$ tablet, Delta Pharma, Cairo, Egypt) are purchased from local sources. NBS and NBP are obtained as reagent grade chemicals form (Alfa Aesar).

All chemicals used are HPLC analytical reagent grade; methanol, acetonitrile (Lab Scan Analytical Sciences), ortho-phosphoric acid (Fisher, USA), sulfuric, hydrochloric, acetic, perchloric acids $\mathrm{BDH}$ (UK) and sodium hydroxide (Loba Chemie). Double distilled water is used for preparing solutions and cleaning all glass equipment. HPLC method is applied using a mobile phase consisting of acetonitrile: $\mathrm{H}_{2} \mathrm{O}$ : phosphoric acid in ratio of $(70: 30: 0.1 \mathrm{v} / \mathrm{v} / \mathrm{v})$, this mixture is filtered and degassed before application.

HPLC analysis is conducted using Agilent 1200 which is equipped with quaternary pump, variable wavelength detector (UV/VIS Detector), Agilent 1200 series vacuum degasser and a manual injector $20 \mu \mathrm{L}$ loop. The compounds are measured on a $150 \times 4.6 \mathrm{~mm} \mathrm{C}_{18}$ column (Agilent-Eclipse XDB $5 \mu \mathrm{m}$ particle size) that is conditioned at $25 \pm 5^{\circ} \mathrm{C}$ with an injection volume of $20 \mu \mathrm{L}$. Potentiometric titrations are carried out using a JENWAY $\mathrm{pH} / \mathrm{mV}$-meter (Model 4510) with a combined platinum-saturated calomel electrode.

Preparation of standard solutions: Standard solutions of Meloxicam $1 \times 10^{-3} \mathrm{~mol}$. $\mathrm{L}^{-1}$ for potentiometric method (I) and $1 \mathrm{mg} \mathrm{mL}^{-1}$ for HPLC method (II) are prepared by dissolving the required amount of drug in $50: 50(\% \mathrm{v} / \mathrm{v})$ methanol: $0.5 \mathrm{~N} \mathrm{NaOH}$ solution, sonicated for a minimum of $20 \mathrm{~min}$ and the solutions are filtered. For HPLC method (II), working standard solution of $\mathrm{Mel}\left(0.1 \mathrm{mg} \mathrm{mL}^{-1}\right)$ is prepared by diluting $5 \mathrm{~mL}$ from $1 \mathrm{mg} \mathrm{mL}^{-1}$ standard solution into $50 \mathrm{~mL}$ volumetric flask with methanol. NBS solution $\left(2 \times 10^{-3} \mathrm{M}\right)$ is prepared by dissolving $17.79 \mathrm{mg}$ of NBS in $50 \mathrm{~mL}$ water while $\left(2 \times 10^{-3} \mathrm{M}\right) \mathrm{NBP}$ solution was prepared by dissolving $23.09 \mathrm{mg}$ of NBP in $25 \mathrm{~mL}$ acetic acid and, after mixing, $25 \mathrm{~mL}$ of water are added to the $50 \mathrm{~mL}$ mark and were standardized according to (Barakat and El-Wahab, 1954). These solutions are stored in amber colored bottles and refrigerated at $4^{\circ} \mathrm{C}$.

Preparation of marketed formulations: To eliminate the inconsistency of weight, five tablets of Melocam or Mexicam are crushed and ground to powdered in an agate mortar. $1 \times 10^{-3} \mathrm{~mol} \mathrm{~L}^{-1}$ and $1 \mathrm{mg} \mathrm{mL}^{-1}$ are prepared as under (Preparation of Standard Solutions). For HPLC method, $5 \mathrm{~mL}$ exactly measured of the filtrate into $50 \mathrm{~mL}$ volumetric flask are transferred and diluted to volume with methanol to get final concentration of $0.1 \mathrm{mg} \mathrm{mL}$.

\section{Analytical method validation}

Potentiometric Method (I): $0.5-4.0 \mathrm{~mL}$ of the standard Meloxicam solution $\left(1 \times 10^{-3} \mathrm{M}\right)$ are accurately measured and transferred into $50 \mathrm{~mL}$ volumetric flask. Each solution is made acidic by adding $1 \mathrm{~mL}$ of $20 \% \mathrm{HCl}$ or $20 \%$ $\mathrm{CH}_{3} \mathrm{COOH}$ ) for NBS and NBP, respectively, after mixing, it is completed to the $50 \mathrm{~mL}$ mark with $\mathrm{H}_{2} \mathrm{O}$. The content of these solutions is titrated with $2 \times 10^{-3} \mathrm{M} \mathrm{NBS}$ or NBP, respectively and the amount of NBS or NBP solution equivalent to the reacted drug amount is calculated.

HPLC method(II): Aliquots of standard Meloxicam solution ( $\left.1 \mathrm{mg} \mathrm{mL}^{-1}\right)$ are taken in calibrated flasks and are made up to the volume with methanol to obtain the concentration range of $5-200 \mu \mathrm{g} \mathrm{mL} \mathrm{m}^{-1}$. The linearity experiment is carried out in triplicates to ascertain accuracy and precision of the method. Samples are injected at intervals of 3 min apart of each concentration and the average peak areas are calculated then plotted versus drug concentrations. Statistical and regression analysis of the calibration data are recorded and fitted to the equation $y=a+b x$. In order to evaluate the applicability of the method to pharmaceutical preparations, the flow rate is set at $2 \mathrm{~mL} \mathrm{~min}^{-1}$ and the detection wavelength at $360 \mathrm{~nm}$. The drug is measured on a $150 \times 4.6 \mathrm{~mm} \mathrm{C}_{18}$ column (Agilent-Eclipse XDB $5 \mu \mathrm{m}$ particle size) that is conditioned at $25 \pm 5^{\circ} \mathrm{C}$. the injection volume is $20 \mu \mathrm{L}$. 
The above methods are applied to the analysis of Mel containing tablets.

\section{RESULTS AND DISCUSSION}

Potentiometric methods (I): Taking advantage of the rapid oxidation and bromination reaction of NBS and NBP with different aromatic compounds (Mathur and Narang, 1975), a quantitative and accurate potentiometric method is developed for the determination of the studied drug. A study of the optimum conditions is carried out and the stoichiometry of the reaction of the Mel with NBS or NBP is ascertained in acidic medium. The conditions optimized are the choice of medium for a quantitative reaction to proceed towards completion and the amount of reagent added.

Effect of acids: Figure 2 shows the effect of using different acid media (hydrochloric, sulphuric, acetic, perchloric, acetic and phosphoric acid) upon the reaction between the drug and NBP (as an example), using a suitable ratio [drug]:[reagent] of 1:2. The data indicates that reproducible and stoichiometric results are obtained using $\mathrm{HCl}$ and $\mathrm{CH}_{3} \mathrm{COOH}$ as medium for the $\mathrm{Mel}$ reaction with NBS and NBP reagent, respectively. As shown in the figure, a sharp inflection lying in the immediate vicinity of the expected end point using hydrochloric and acetic acid more than the other acids is found in the case of NBS and NBP, respectively. So, hydrochloric and acetic acid are preferred for carrying out the potentiometric titration of Mel using NBS and NBP, respectively.

Also, the results obtained reveals that the end point is unaffected by the addition of (1-3 mL) of any acid used as shown in Fig. 3. However, it is observed that the use of $1 \mathrm{~mL}$ from $20 \% \mathrm{v} / \mathrm{v} \mathrm{HCl}$ or $\mathrm{CH}_{3} \mathrm{COOH}$ causes a sharp inflection at the expected end point for NBS and NBP, respectively. Thus, $1 \mathrm{~mL} \mathrm{HCl}$ or $\mathrm{CH}_{3} \mathrm{COOH}$ is maintained during the current investigations.

Effect of NBS and NBP reagents: For the quantitative determination of Mel, the effect of NBS and NBP concentration is tried and $2 \times 10^{-2} \mathrm{M}$, is chosen as an optimum concentration to achieve a constant and highly stable potential reading during the mixing time. Concentration less than this leads to unstable readings and more time is needed to obtain constant values.

Also arising temperature does not accelerate the oxidation process but tends to cause inaccurate results and creates difficulty in detecting the end point, because NBS or NBP decomposes at high temperature, thus room temperature is the most suitable.

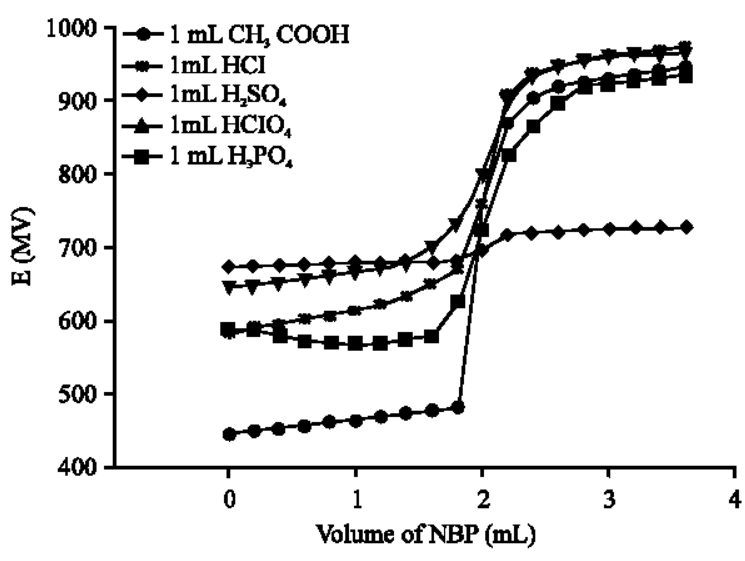

Fig. 2: The effect of acids on the titration of 2 Meloxicam with NBP reagent [Meloxicam] $=1 \times 10^{-3} \mathrm{M}$, $[\mathrm{NBP}]=2 \times 10^{-3} \mathrm{M}$ and $[$ Acid $]=20 \% \mathrm{v} / \mathrm{v}$

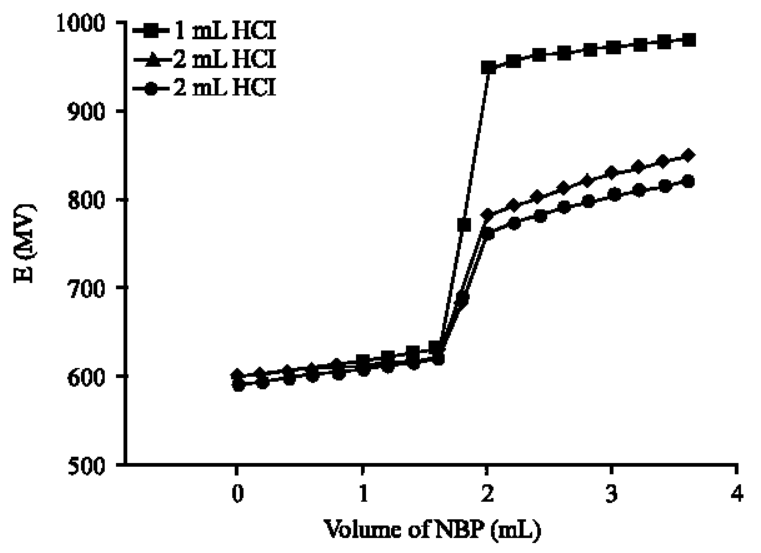

Fig. 3: The effect of HCL concentration on the titration of Meloxicam with NBS reagent [Meloxicam] $=1 \times 10^{-3}$ $\mathrm{M},[\mathrm{NBS}]=2 \times 10^{-3} \mathrm{M}$ and $[$ Acid $]=20 \% \mathrm{v} / \mathrm{v}$

Based on the obtained experimental results and the chemical property of Mel, NBS and NBP, the reaction between Mel with each of NBS and NBP may take place via the attack on the respective $\mathrm{NH}$ and $\mathrm{OH}$ groups of the drug (Mathur and Narang, 1975). The first molecule of NBS or NBP may be consumed in the bromination of Mel $\mathrm{NH}$ group while the second one in the bromination of $\mathrm{OH}$ group or vice versa, to give the dibromo derivative of the drug as previously reported (El-Ries et al., 2003).

Potentiometric titration of meloxicam: The reaction is found to be quantitative with a stoichiometry of $1: 2$ (Mel: NBS or Mel: NBP) for the investigated concentration range of $0.176-1.406 \mathrm{mg} \mathrm{Mel}$ for NBS and NBP, in hydrochloric and acetic acid, respectively. The titration curves of drug show one well-defined S-shaped 


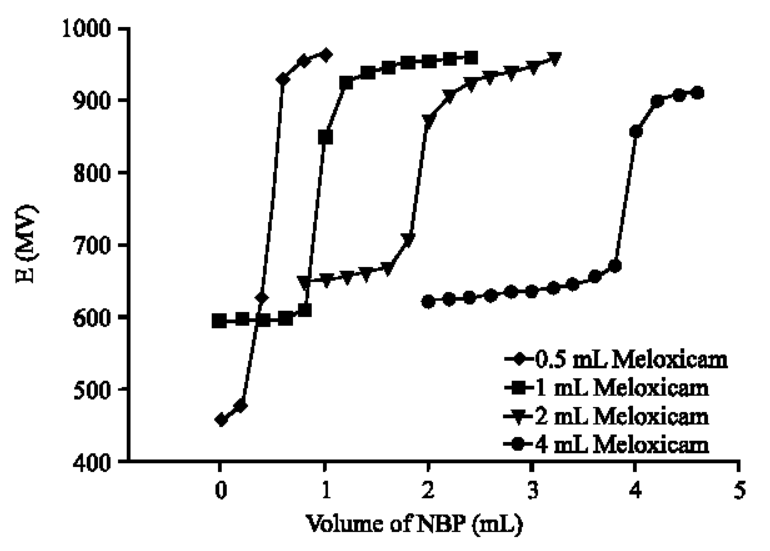

Fig. 4: Typical titration curves for potentiometirc determination of the end point of different concentrations of Meloxicam, against NBS reagent. $[$ Meloxicam $]=1 \times 10^{-3} \mathrm{M}$ and $[\mathrm{NBS}]=2 \times 10^{-3} \mathrm{M}$

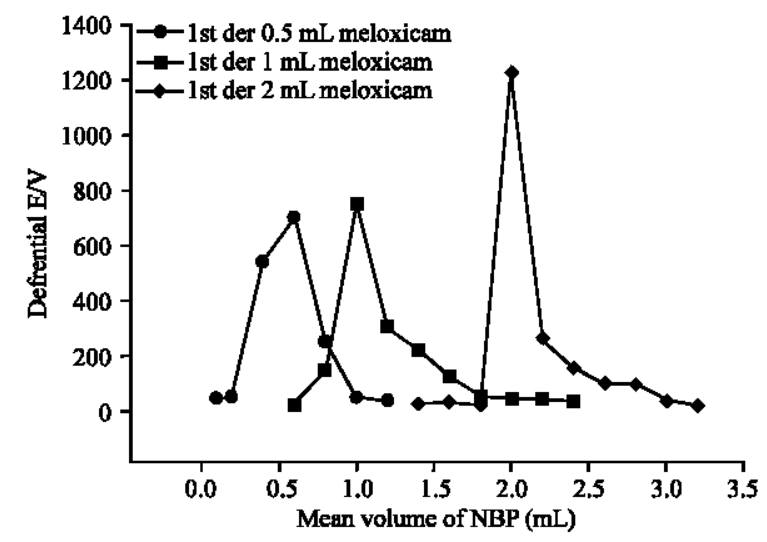

Fig. 5: Differential curves for the potentiometirc titration of different concentrations of Meloxicam against NBP reagent. [Meloxicam] $=1 \times 10^{-3} \mathrm{M}$ and $[\mathrm{NBP}]=2 \times 10^{-3} \mathrm{M}$

stoichiometric end point using a Pt-electrode as an indicator electrode. The titration data is given in Fig. 4 and 5 for NBS and NBP, respectively. Figure 4 represents the plot of $\mathrm{E}$ vs. volume (mL) of NBS titrant for the determination of Meloxicam The first differential curves which represent the plot of $\Delta \mathrm{E} / \Delta \mathrm{V}$ vs., mean volume of NBP titrant are characterized by sharp inflections lying in the immediate vicinity of the expected end points (Fig. 5). These sharp inflections permit the accuracy of the end point which indicate that NBS and $\mathrm{NBP}$ can be used as reagents for a sensitive determination of Meloxicam.

\section{Analytical parameters}

Potentiometric method: To ascertain the accuracy and precision of the proposed methods, three replicate
Table 1: Summary of validation data for the proposed methods for the analysis of Meloxicam in bulk solution

\begin{tabular}{llll}
\hline & Methods & & \\
\cline { 2 - 4 } & NBC reagent & NBP reagent & HPLC method \\
\hline Parameters & $0.176-1.406 \mathrm{mg}$ & $0.176-1.406 \mathrm{mg}$ & $5-200 \mu \mathrm{g}$ \\
Range of Concentration & $1.000^{\mathrm{a}}$ & $0.998^{\mathrm{a}}$ & 0.999 \\
Correlation Coefficient & $1.006^{\mathrm{a}}$ & $1.007^{\mathrm{a}}$ & 66.021 \\
Slope & $0.002^{\mathrm{a}}$ & $0.001^{\mathrm{a}}$ & 67.13 \\
Intercept & 3 & 3 & 3 \\
$\mathrm{~N}$ & $100.26 \pm 0.73$ & $100.08 \pm 0.68$ & $99.41 \pm 0.49 \%$ \\
Mean $\pm \mathrm{RSD} \%$ & 0.73 & 0.68 & 0.49 \\
$\mathrm{SD}$ & 0.42 & 0.39 & 0.28 \\
SE & & & \\
\hline a Observed vs, theoretical values &
\end{tabular}

determinations using four different concentration of the drug are performed. The mean recovery values and the Relative Standard Deviation (RSD) are 100.26+0.73 and $100.08+0.68 \%$ for NBS and NBP, respectively and the standard error are presented in Table 1. These data indicate high accuracy and precision of the methods. In order to establish whether the proposed potentiometric methods exhibited any fixed or proportional bias, a simple linear regression (Miller and Miller, 1993) of the observed drug concentration against the theoretical values is obtained. The slope and intercept of the regression line was 1.006 and 0.002 for NBS and 1.007 and 0.001 for NBP while the correlation coefficient is 1.000 and 0.999 for NBS and NBP, respectively.

HPLC method: The experiment is applied to various parameters such as efficiency resolution and peak symmetry. In order to avoid ion suppression induced by endogenous substances, the mobile phase for the reaction is selected after several trials with acetonitrile, water and phosphoric acid in various ratios and at various pHs. However, the optimal mobile phase is found to be consisting of a mixture of $70: 30: 0.1(\mathrm{v} / \mathrm{v} / \mathrm{v})$ acetonitrile: $\mathrm{H}_{2} \mathrm{O}$ : phosphoric acid.

Meanwhile, under the present chromatographic conditions, the mobile phase provides good linearity for the studied concentration, the run time between each sample is $3 \mathrm{~min}$ and the retention time is $2.1 \mathrm{~min}$ for the drug.

For quantitative analysis using HPLC method, the linearity experiment is carried out in triplicates to ascertain its accuracy and precision. The method exhibits a good linear response for the range of concentrations from 5-200 $\mu \mathrm{g} \mathrm{mL}-1$ with coefficient of determination 0.999 . Typical standard curve is $\left[\mathrm{y}=66.0 \mathrm{x} .+67.13, \mathrm{r}^{2}=0.999\right]$ where $\mathrm{y}$ is the area and $\mathrm{x}$ is the concentration of the drug. While LOD/LOQ parameters are not a requirement for drug assay; it is always useful to demonstrate that the analysis is being conducted in a region which is above the $L O Q$ value. The limits of detection $(\mathrm{LOD}=3 \mathrm{SD} / \mathrm{b})$ and 
quantification $(\mathrm{LOQ}=10 \mathrm{SD} / \mathrm{b})$ are calculated according to ICH (Aeri et al., 2010). The values are derived from the Standard Deviation of the response (SD) and the slope of calibration curves (b) and are found to be 0.04 and 0.14 , respectively.

The intra-day accuracy and precision of the assay is evaluated by analyzing nine samples of the drug at concentrations $\left(80,100\right.$ and $\left.150 \mu \mathrm{g} \mathrm{mL}^{-1}\right)$ and was found to ranged from $99.87-102.02$ and $0.76-1.12 \%$, respectively. The inter-day accuracy and precision is determined over three days. The inter-day accuracy range from 98.21-100.08\% while relative standard deviation obtained are ranged from $0.84-1.3 \%$.

Stability of the standard solution: For short-term stability determination, standard solution is monitored by analyzing standard solution aged at ambient temperature and ambient light against freshly prepared standards. Samples are pretreated and analyzed as abovementioned. The mean recoveries ranges from 99.89-100.01\%. These results indicate reliable stability behavior under the experimental conditions.

The ruggedness of the method is also proved by using two chromatographic columns from two different suppliers. The mean percentage found $(n=9)$ were 99.57 and $99.47 \%$ while the precision is 1.45 and $0.53 \%$ for Agilent-Eclipse XDB $\mathrm{C}_{18} 150 \times 4.6 \mathrm{~mm}$, 5um and Luna-Phenomenex $\mathrm{C}_{18} 150 \times 4.6 \mathrm{~mm}, 5$ um column, respectively.

Application to pharmaceutical formulations: Specificity is the ability to assess clearly the analyte in the presence of components which may be expected to be present. The proposed methods (potentiometric and HPLC) are applied to the analysis of pharmaceutical formulations of the drug (Melocam and Mexicam tablets). The fact that the shapes of the potentiometric titration curves of bulk drug and its formulations are nearly the same, proves that the excipients which may be present in the pharmaceutical formulations has no effect on the determination of the drug applying the proposed method.

As shown in Fig 6 and 7, the HPLC chromatogram of bulk Meloxicam is nearly the same as for Melocam tablet without any considerable changes in retention time, so it recommends that the proposed HPLC method is suitable for determination of the drug.

The results of the assay of meloxicam in pharmaceutical formulations are compared with those obtained by the official method (USP, 2009). Statistical comparison of the results is performed with regard to accuracy and precision using the Student's t-test and F-tests (Table 2). At 95\% confidence level, the calculated

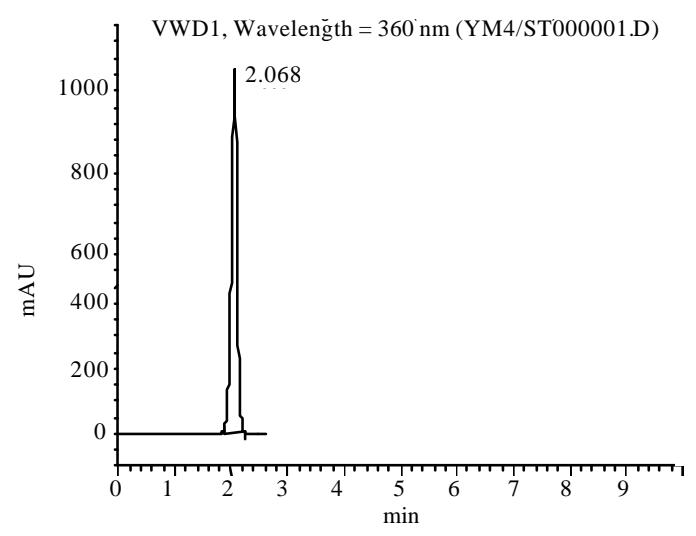

Fig. 6: Chromatogram of meloxicam

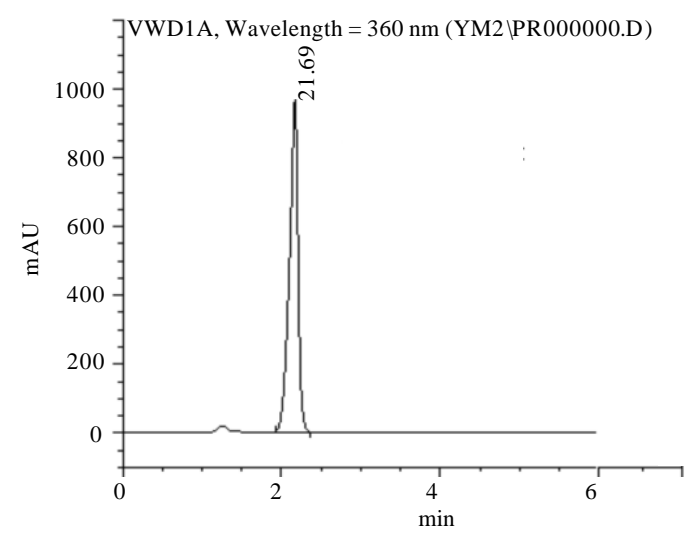

Fig. 7: Chromatogram of melocam tablets

t-and F-values does not exceed the theoretical values, indicating that there is no significant difference between the proposed methods and the official method with regard to accuracy and precision. The data obtained also shows good recoveries and low standard deviations.

The recovery studies of standard additions to commercial pharmaceuticals are also carried out to provide further support for the validity of the method. The related data are given in Table 3 . These results also confirm the precision and the validity of the proposed method.

In pharmaceutical formulations analysis, it is necessary to test the selectivity towards excipients and fillers added to pharmaceutical preparations. The proposed methods are applied to the analysis of Meloxicam in tablets and the results are compared with those obtained by the official method. Commonly encountered excipients such as starch, talc, glucose, alginate and stearate are not interfere with the proposed methods. Statistically, Table 2 indicates that the 
Insight Pharmaceutical Sciences 2 (1): 1-7, 2012

Table 2: Determination of Meloxicam in some pharmaceutical preparations apply ing the proposed methods

\begin{tabular}{|c|c|c|c|c|c|}
\hline \multirow[b]{3}{*}{ Method } & \multicolumn{5}{|l|}{ Recovery\%+SD } \\
\hline & \multicolumn{2}{|l|}{ Melocam tablets } & & \multicolumn{2}{|l|}{ Mexicam tablets } \\
\hline & $(7.5 \mathrm{mg})$ & \multicolumn{2}{|l|}{$(15 \mathrm{mg})$} & $(7.5 \mathrm{mg})$ & $(15 \mathrm{mg})$ \\
\hline NBS Mean Recovery & $100.74+0.60$ & \multicolumn{2}{|l|}{$99.91+0.46$} & $98.99+0.21$ & $100.04+0.38$ \\
\hline $\mathrm{N}$ & 3 & \multicolumn{2}{|l|}{3} & & 3 \\
\hline SD & 0.60 & \multicolumn{2}{|l|}{0.46} & 0.21 & 0.38 \\
\hline $\mathrm{SE}$ & 0.34 & \multicolumn{2}{|l|}{0.26} & 0.12 & 0.22 \\
\hline t-value & 1.94 & \multirow{2}{*}{\multicolumn{2}{|c|}{1.94}} & 1.94 & 1.94 \\
\hline F-value & 4.29 & & & 1.91 & 1.72 \\
\hline NBP Mean Recovery & $99.96+0.18$ & \multicolumn{2}{|l|}{$100.06+0.32$} & $100.13+0.77$ & $99.96+0.98$ \\
\hline $\mathrm{SD}$ & 0.18 & 0.32 & & 0.77 & 0.98 \\
\hline SE & 0.10 & 0.18 & & 0.44 & 0.56 \\
\hline t-value & 0.86 & 0.86 & & 0.86 & 0.86 \\
\hline F-value & 2.59 & 1.22 & & 7.06 & 11.43 \\
\hline HPLC & $100.10 \pm 1.09$ & $100.01 \pm 0.91$ & & $99.89 \pm 0.59$ & $99.12 \pm 0.49$ \\
\hline SD & 1.09 & 1.09 & & 1.09 & 1.09 \\
\hline $\mathrm{SE}$ & 0.63 & 0.53 & & 0.34 & 0.28 \\
\hline t-value & 0.18 & 0.04 & & 0.26 & 2.66 . \\
\hline F-value & 14.14 & 9.86 & & 4.14 & 2.86 \\
\hline Official $^{(2)} \mathrm{HPLC}$ method & $99.99 \pm 0.29$ & & & & \\
\hline The tabulated values of $\mathrm{t}-$ an & ts at $95 \%$ confidenc & 7) and (19), respecti & & & \\
\hline Pharmaceutical preparation & Reagent & Taken (mg) & Added (mg) & Found (mg) & Recovery \\
\hline Melocam tablets (15mg) & NBS & 0.3 & 0.2 & 0.498 & 99.6 \\
\hline & & 0.3 & 0.4 & 0.710 & 101.4 \\
\hline & & 0.3 & 0.6 & 0.903 & 100.3 \\
\hline & & 0.3 & 0.8 & 1.079 & 98.1 \\
\hline Average & & & & & 99.85 \\
\hline RSD\% & & & & & 1.221 \\
\hline Mexicam tablets (15mg) & NBP & 0.4 & 0.2 & 0.611 & 101.833 \\
\hline & & 0.4 & 0.4 & 0.802 & 100.250 \\
\hline & & 0.4 & 0.6 & 1.01 & 101.000 \\
\hline & & 0.4 & 0.8 & 1.197 & 99.750 \\
\hline Average & & & & & 100.708 \\
\hline RSD\% & & & & & 0.67 \\
\hline
\end{tabular}

calculated values of $\mathrm{t}$ and $\mathrm{F}$ test do not exceed the theoretical values. Therefore, there is no significant difference in the mean recoveries and precision between the proposed and official method.

\section{CONCLUSION}

In conclusion, the described methods are suitable for the determination of Meloxicam in pure form and in formulated products. The proposed potentiometric method have some specific advantages, that its oxidants are the most effective and stable brominating agent colorless, easily available, less expensive, provided higher sensitivity and they have the ability of being dissolved in the aqueous and non-aqueous solvents.

The proposed HPLC method is precise and accurate, selective, in addition, the mobile phase of actonitrile, $\mathrm{H}_{2} \mathrm{O}$ and acetic acid in the ratio of 70: $30: 0.1(\mathrm{v} / \mathrm{v} / \mathrm{v})$ without the need to adjust the $\mathrm{pH}$, is found to be ideal for the analysis of $\mathrm{Mel}$.

The experimental data are considered satisfactory and indicate specificity, linearity, accuracy, repeatability and sensitivity of the developed methods. These results indicate that these methods are suitable for routine quality control analysis.

\section{REFERENCES}

Acuña, J.A., M.D. Vázquez, M.L. Tascón and P. Sánchez-Batanero, 2004. Polarographic behaviour of aceclofenac, tenoxicam and droxicam in a methanol water mixture. J. Pharm. Biomed. Anal., 36: 157-162.

Aeri, Vidhu, M.I. Khan and S. Alam, 2010. A validated HPLC method for the quantification of oleanolic acid in the roots of achyranthes aspera linn. and marketed formulation. Int. J. Pharm. Pharmaceut. Sci., 2: 74-78.

Al-Kindy, S.M.Z., F.E.O. Suliman, S. Al-Habsy and H. Al-Lawati, 2010. Determination of meloxicam and flufenamic acid in pharmaceutical formulations and biological fluids using lanthanide sensitized luminescence. Proceedings of the International Conference and Exhibition on Analytical and Bioanalytical Techniques, Nov. 1-3, Merriott Hotel and Convention Center, Hyderabad, India. 
Al-Nimer, M.S.M. and E.A. Ali, 2009. Dual effectsof interaction between meloxicam, diclofenac sodium or tramadol and nitrogen species radicals: In vitro comparative study. Int. J. Pharmacol., 5: 86-89.

Bandarkar, F.S. and P.R. Vavia, 2009. A stability indicating HPLC method for the determination of meloxicam in bulk and commercial formulations. Trop. J. Pharmaceut. Res., 8: 257-264.

Barakat, M.Z. and A.M.F. El-Wahab, 1954. Micro-determination of bromine or chlorine in N-halogenated imides. Anal. Chem., 26: 1973-1974.

Cheng, W.Y., Z.X. Wang, J. Guan and X.Y. Hu, 2006. Voltammetric determination of meloxicam in pharmaceutical formulation and human serum at glassy carbon electrode modified by cysteic acid formed by electrochemical oxidation of 1-cysteine. Sensors, 6: 1139-1152.

Desai, N. and P. Amin, 2008. Stability indicating HPTLC determination of meloxicam. Indian J. Pharm. Sci., 70: 644-647.

Dhandapani, B., S.E. Murali, N. Susrutha, R. Swetha and S.K.S. Rani et al., 2010. Spectrophotometric estimation of meloxicam in bulk and its pharmaceutical formulations. Int. J. Pharma. Sci. Resea., 4: 217-221.

El-Ries, M.A., G. Mohamed, S. Khalil and M. El-Shall, 2003. Spectrophotometric and potentiometric determination of piroxicam and tenoxicam in pharmaceutical preparations. Chem. Pharm. Bull., 51: $6-10$.

Erğlu, H., N. Burul-Bozkur, S. Uma and L. Öner, 2010. Validation of the analytical method for in-vivo determination of meloxicam and bioequivalence study from meloxicam containing microparticle formulations in rabbits. Hacettepe University J. Facul. Pharmacy, 2: 115-130.

Farhadi, K. and A. Karimpour, 2007. Electrochemical determination of meloxicam in pharmaceutical preparation and biological fluids using oxidized glassy carbon electrodes. Chem. Pharm. Bull., 55: 638-642.

Huazhen, Y., B. Qiu, J. Chen, J. Lin and G. Chen, 2009. Flew-injection analysis for meloxicam based on tris $(2,2$ '-bipyridine) ruthenium(II)-CE(IV) chemiluminescent system. Luminescence, 24: $260-265$.

Jan, K. and M. Starek, 2009. A review of analytical techniques for determination of oxicams, nimesulide and nabumetone. Talanta, 77: 925-942.
Kumar, K.G. and P. Indrasenan, 1988. Titrimetric methods for the determination of some sulpha drugs using N-bromophthalimide and N-bromosaccharin. Analyst, 113: 1369-1372.

Lee, H.W., H.Y. Ji, H. Y. Kim, K.C. Lee and H.S. Lee, 2009. Liquid chromatography-tandem mass spectrometry method for the determination of meloxicam and its metabolite 5-carboxymeloxicam in human plasma. Bioanalysis, 1: 63-70.

Liu, H., L. Zhang, Y. Hao, Q. Wang, P. He and Y. Fa, 2005. Flow-injection chemiluminescence determination of meloxicam by oxidation with N-bromosuccinimide. Anal. Chim. Acta, 541: 185-190.

Mahmood, K.T., B. Khan, M. Ashraf and I.U. Haq, 2010. Specific and simple HPLC Assay of ecofriendly meloxicam in pharmaceutical formulations. J. Pharm. Sci. Res., 12: 878-883.

Martindale, 2002. The Extra Pharmacopoeia. 33rd Edn., Pharmaceutical Press, London, UK.

Mathur, N.K. and C.K. Narang, 1975. The Determination of Organic Compounds With N-Bromosuccinimide and Allied Reagents. 1st Edn., Academic Press, London, pp: 54-59.

Miller, J.C. and J.N. Miller, 1993. Statistics for Analytical Chemistry. 1st Edn., Ellis Horwood PTR Prentice-Hall New York.

Radia, O. and G. Moulay-Hassan, 2010. Liquid chromatographic determination of meloxicam in serum after solid phase extraction. Chemical Papers, 64: 429-433.

Sinha, P.K., R.M. Jeswani, K.S. Topagi and M.C. DamLe, 2009. A validated RP-HPLC method for determination of meloxicam in the presence of its impurities. Int. J. Pharm. Tech. Res., 1: 1051-1060.

Thanh, T.D., J. Flores-Jaime, C. Alvarez-Alvares and P.M. de la Torre-Iglesias, 2010. Spectrophotometric simultaneous determination of meloxicam and sodium amoxicillin in combined dosage forms. Pharm. Res., 3: 425-428.

USP, 2009. The United States Pharmacopoeia Convention. USP, Rockville, MD.

Yuan, Y., X. Chen and D. Zhong, 2007. Determination of meloxicam in human plasma by liquid chromatography-tandem mass spectrometry following transdermal administration. J. Chromat. B. Analyt Technol. Biomed Life Sci., 2: 650-654. 